\title{
Editorial
}

\section{Change Management}

\section{Associate Editors Gillian Jack and Kathy Whymark}

The Editors are pleased to offer this opportunity for the associate editors for Change Management to outline their domain, and seek contributions from potential authors.

Approaches to change tend to appear as waves, with various theorists introducing their own perspectives. Changes include those in society that influence organisations, and these in turn include, for example, more flexible working environments, growth of women in management, increased levels of education amongst the workforce, and views that people are assets that should be nurtured. Indeed, the latter view is a major factor in the drive to consider intellectual assets explicitly. Diverse working patterns, complexity of cultures and speed of change in large organisations, mean that social stability and appropriate human resource management are emerging as major strategic challenges.

The emergence of the concept of the learning organisation and knowledge management initiatives mean that the skills and knowledge that managers need in order to manage change effectively are contextually and culturally diverse, and include issues relating to political, economic, social, and technological influences. To manage change strategically requires both a rational approach, and an ability to address successfully the formal and informal power relationships between people, their attitudes to change, their values and beliefs, the culture of the organisation, the status of relationships, and the morale of staff. Fairly straightforward then!

From the above it can be deduced that change addresses cross-organisational development to achieve longer-term objectives based on competitiveness, successful performance, and market position. A changeable external and internal environment that can interrupt, delay, speed up or terminate progress influences this. To introduce a common sense of purpose and a persuasive rationale managers should not underestimate the need to involve the broader workforce.

The foregoing gives a general overview of the area, but potential contributors should not feel constrained to accept this view. Papers on any area of change are welcome, and the following are suggestions that are intended to assist, rather than set out predefined, authoritative, and definitive limitations.

- Case studies or action research discussing approaches to organisational change in practice.

- Issues of organisational behaviour and wellbeing in the context of change.

- Reports of successes or failures resulting from the use of change methodologies.

- The relationships between people, organisations, information technology and managing change.

- New approaches to Human Resource Management.

- Cross cultural change management.

- Specific case studies relating to change and conflict in organisations.

- Organisational change in a social context.

When submitting your paper, please remember to address the general aims of OR Insight, which can be found on the back page, together with formatting instructions.

Please send any electronic copy of papers on organisational change to Gillian Jack or Kathy Whymark, via gillian.jack@luton.ac.uk or kathy.whymark@luton.ac.uk.

\section{We look forward to receiving your contributions.}

